\title{
Analysis of below-knee suspension systems: Effect on gait
}

\author{
Roy W. Wirta, BSME; Frank L. Golbranson, MD; Randy Mason, CP; Kevin Calvo, CP \\ VA Medical Center, San Diego, CA 92161
}

\begin{abstract}
Seven different suspension systems for below-knee prostheses were tested on 20 adult, unilateral amputees. All subjects walked with a prosthesis. Gait was evaluated with regard to longitudinal and vertical accelerations of the body, forward velocity, axial (piston) movement of amputated limb in the socket, and knee movement. Harmonic analyses of variable waveforms provided the primary method to discriminate performances among the suspensions. The appropriateness of a given suspension system depended on both the length and the shape of the residual limb.
\end{abstract}

Key words: below-knee prostheses, gait analysis, residual limb, socket, stump, suspension systems, unilateral amputees.

\section{INTRODUCTION}

Numerous means to suspend below-knee (BK) prostheses have been developed (9). Diversity has resulted from attempts to fit individuals of differing physical characteristics and life styles. Although suspension systems generally used for BK prostheses have similar functions, choices for

Address all correspondence and requests for reprints to: Roy W. Wirta, BSME, 5570 Rab Street, La Mesa, CA 91942.

This work was supported by a grant entitled, "Analysis of Below-Knee Suspension Systems: Effect on Gait," from the Department of Veterans Affairs Rehabilitation Research and Development Service and was conducted at the VA Medical Center, San Diego, CA 92161.

Mr. Wirta and Dr. Golbranson have retired from the VA Medical Center at San Diego. They may be reached at the following home addresses: Roy W. Wirta, BSME, 5570 Rab Street, La Mesa, CA 92042; Frank L. Golbranson, MD, 6201 Camino De La Costa, La Jolla, CA 92037. Other addresses: Randy Mason, CP, Orthotic-Prosthetic Associates, 3665 Kearny Villa Road, Suite 360, San Diego, CA 92123; Kevin Calvo, CP, Bionics Orthotics and Prosthetics, 3737 Moraga Avenue, Suite 220, San Diego, CA 92117. prescription are not totally clear. Patellar tendon bearing (PTB) suspension (10) principles are effective with many patients; however, those with short limbs may not achieve complication-free suspension. The fact that variations of $\mathrm{BK}$ suspensions continue to appear suggests that a universal suspension system is not yet available.

The main purpose of all suspension systems is prosthesis retention; however, the effects of other aspects of limb-socket biomechanics are not obvious (e.g., certain suspension systems, designed to minimize axial socket movement (AXM), sometimes alter the biomechanical effect of the prosthesis). Some factors, such as restriction of knee excursion, alter the gait pattern and efficiency.

This study was designed to measure the effects on gait of seven different commonly prescribed BK suspension systems, and to relate these effects to the length and shape of the residual limb in order to make prescription criteria more explicit.

\section{DESCRIPTION OF SUSPENSION SYSTEMS}

\section{PTB with suprapatellar cuff and/or waistbelt}

The suprapatellar cuff was an integral part of the PTB prosthesis as described by Radcliffe and Foort in 1961 (10). This is probably the most widely used type of suspension and is usually adequate for most wearers. The cuff loosens during knee flexion and tightens during extension. An optional waistbelt augments suspension during the swing phase; however, the waistbelt is unacceptable to many patients. 
Journal of Rehabilitation Research and Development Vol. 27 No. 4 Fall 1990

\section{PTB with figure-of-eight}

The figure-of-eight system is a variation of the suprapatellar cuff as introduced and described in 1972 by Girling and Cummings (6). This continuous strap must be tight in order to be effective, which can lead to vascular constriction as well as bunching of posterior soft tissues.

\section{Rubber sleeve}

This system has been used extensively at the University of Michigan since 1968. It affords good suspension for the patient who has substantial soft tissue where suspension over bony prominences is made difficult with other types of systems. The original latex sleeves are difficult to don and can restrict knee movement, retain heat, and foster perspiration under the sleeve.

\section{Supracondylar-suprapatellar}

This type of suspension was introduced into the United States by Marschall and Nitschke in 1966 (7). It has traditionally been linked with the ability to suspend and stabilize the prosthesis, particularly for residual limbs shorter than $11 \mathrm{~cm}$ ( 4.5 inches), as suggested by Breakey (1). The ability for self-suspension is inherent in the design; therefore, it has become a widely used method of treating belowknee amputees. Because of the proximal brim orientation, both mediolateral and anterior stability are enhanced. As the patient begins the forward progression over the foot by extension of the knee, the prosthesis can be timed to start forward when the suprapatellar area contacts the socket. For patients with shorter residual limbs, the overall effect is improved control of the prosthesis. The disadvantages are poor silhouette and loss of suspension when the knee is flexed.

\section{Supracondylar}

This is a variation of the supracondylar-suprapatellar suspension and is used generally with patients who have longer residual limbs and who can better control the prosthesis in the anterior plane. The supracondylar suspension, although adequate, is not without drawbacks; mainly, lack of suspension during high degrees of knee flexion. As the patient flexes the knee, the femoral condyles, which contribute to suspension of the prosthesis, rotate in relation to the suspension wedge, creating less and less surface contact with the wedge. Furthermore, as rotation occurs, the point of suspension becomes more posterior, often coming completely out of the socket during extreme degrees of flexion (but generally not during walking). It is at this point that the suspension is least effective.

\section{Articulated supracondylar wedge}

Because of the lack of suspension during knee flexion by both the suprapatellar wedge and supracondylar-type suspensions, a variation of both was developed. The articulated supracondylar wedge uses the femoral condyle for suspension, but is hinged with the socket at approximately the axis of rotation of the knee. Because of the flexible nature of the material used for the supracondylar wedge (polypropylene), additional medial-lateral (M-L) stability is not gained. To provide adequate range of motion, the medial brim must be trimmed relatively low, thus contraindicating application to patients with residual limbs that require enhanced $\mathrm{M}-\mathrm{L}$ stabilization.

\section{Test subjects}

The selection criteria for serving in this study were that the subjects be adult, unilateral, below-knee amputees who were full-time (community) ambulators, had asymptomatic residual limbs, and did not need auxiliary ambulation aids. The study included both males and females and excluded subjects who were psychiatrically non-compliant or had balance problems.

\section{Suspension systems}

The suspension systems used in this study were considered representative of several commonly used general design concepts for the PTB BK prostheses. These systems were:

1. Supracondylar, suprapatellar (SCSP)

2. Supracondylar (SC)

3. Cuff (PTB/C)

4. Waistband and cuff (PTB/WB)

5. Figure-of-eight suprapatellar strap (PTB/F8)

6. Rubber sleeve (RS)

7. Articulated supracondylar wedge (ASCW).

Each subject was fitted with a PTB socket in accordance with the basic design described by Radcliffe and Foort (10). The prosthesis included a three-sixteenth-inch thick polyethylene foam liner and a SACH foot. Each prosthesis was aligned statically and dynamically with an alignable pylon system.

1. The prosthesis was initially designed as a supracondylar, suprapatellar suspension incorporating the wedges in the insert as outlined by Marschall and Nitschke (8).

2. The first alteration was to trim the suprapatellar component to create the supracondylar suspension.

3. In the second alteration, the supracondylar component was trimmed to achieve the conventional PTB design presented by Radcliffe and Foort (10). A leather supracondylar cuff was applied. 
4. The third alteration was to add a waistbelt and elastic strap to the cuff.

5. In the fourth alteration, the cuff was removed and a figure-of-eight strap was installed. A one-inch wide polyester strap was secured to the medial brim of the socket, forming a figure-of-eight, and attached to the lateral socket with a hook and pile fastener.

6. In the fifth alteration, the straps were removed and a latex sleeve was applied, as described by Giacinto (5).

7. The sixth and final alteration to the prosthesis was the removal of the rubber sleeve and installation of the articulated supracondylar wedge (Figures $\mathbf{1 a}, \mathbf{1 b}$, and 1c). The system was fabricated by taking a negative mold of the subject's knee $5 \mathrm{~cm}$ ( 2 inches) above the adductor tubercle, with the residual limb fully in the socket, and the knee flexed to approximately 40 degrees. A positive mold was then made on which to vacuum-form the polypropylene wedge. The wedge was attached to the medial brim at a point approximately at the axis of knee rotation, and extended from the lateral aspect of the patella medially to the posterior medial corner of the residual limb, just

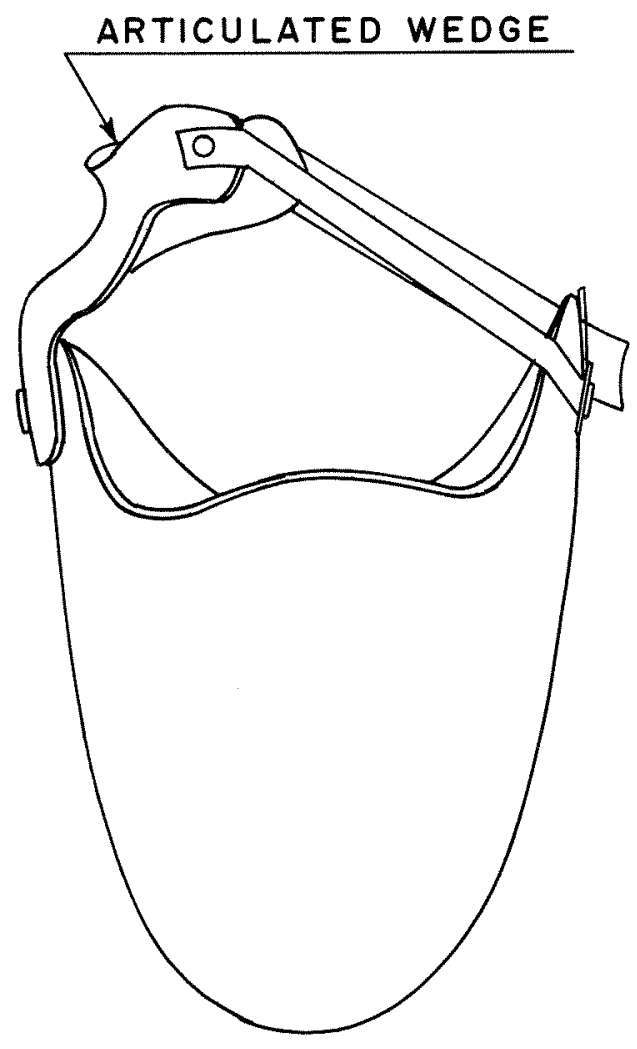

POSTERIOR VIEW

Figure 1a.

Posterior view of articulated supracondylar wedge suspension.
SUPRAPATELLAR STRAP

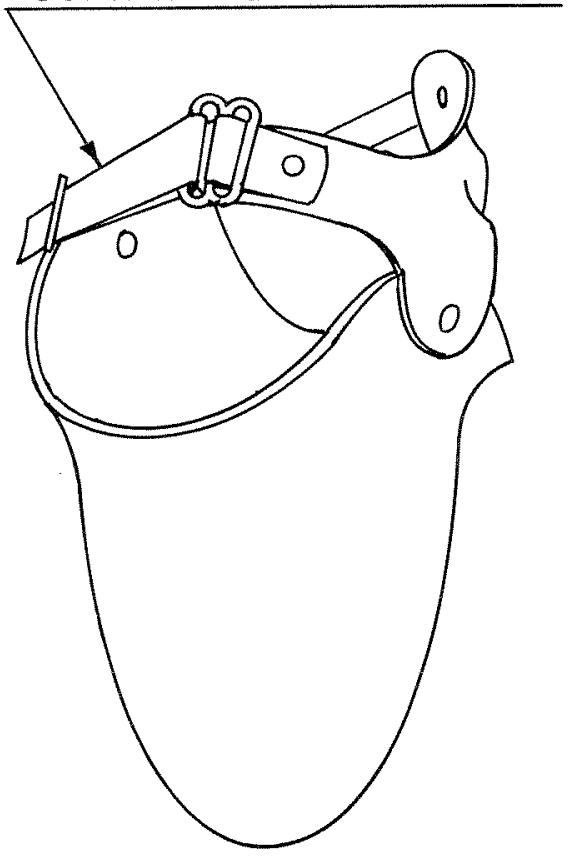

ANTERO-MEDIAL VIEW

Figure 1b.

Antero-lateral view of articulated supracondylar wedge suspension.

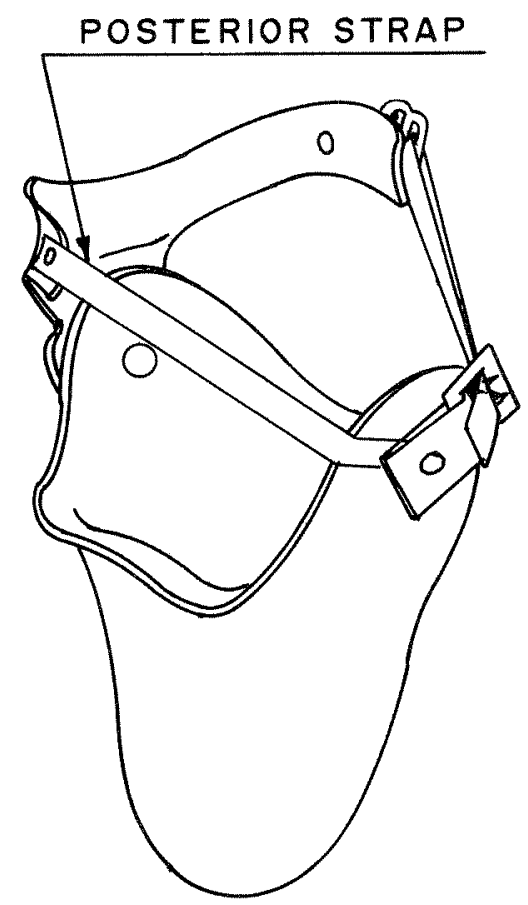

\section{POSTERO-LATERAL VIEW}

Figure 1c.

Postero-lateral view of articulated supracondylar wedge suspension. 
Journal of Rehabilitation Research and Development Vol. 27 No. 4 Fall 1990

proximal to the femoral condyle. The wedge was attached to the lateral brim of the socket by means of: $a$ ) a halfinch wide, fixed length posterior strap; and, $b$ ) a one-inch wide polyester suprapatellar strap that pulled the medial wedge laterally when tightened.

\section{Experimental}

The subjects were attired in shorts, a shirt, and consistent street footwear. Body-mounted instrumentation included bilateral knee electrogoniometers, foot switches, an axial movement detector in the prosthesis, a triaxial accelerometer mounted on a hip-waistbelt, and a lightweight backpack containing an electrical junction box. Two tests were administered with each suspension: the first immediately after a given suspension was fitted, and the second one week later. The subjects were asked to wear the prosthesis for at least 2 hours per day between weekly appointments.

The subjects were instructed to walk (on the walkway) at three different speeds: 1) "usual"; 2) faster than usual; and, 3) slower than usual. Axial movement of the distal aspect of the stump with respect to the socket was monitored with a device installed in a well at the bottom of the socket (Figure 2). A plunger using a force of about onehalf newton drove a potentiometer which followed the relative movement of the distal aspect of the stump. Knee flexion and extension was monitored bilaterally with electrogoniometers attached anteriorly to the tibial and femoral areas. Parallelogram linkages transferred relative limb segment angular movements to potentiometers.

Forward velocity was measured with a tachometer consisting of a low inertia dc generator mounted on a stationary base at one end of the walkway. A thin braided steel cable 0.014-inch in diameter connected the tachometer to a waistband on the subject. A weight-and-pulley arrangement maintained a constant tension of about one newton on the cable. Whole body accelerations were monitored with a miniature triaxial device used in a biaxial mode to register the longitudinal (fore-aft) and vertical axes of motion. The device was mounted in a two-axis gimbal arrangement secured to the sacrolumbar area with a snugfitting hip-waistbelt. The gimbal was stabilized to the direction of forward progression of the body by the tachometer cable through a mechanical filter. Foot switches $1.5 \mathrm{~mm}$ thick were taped to the shoes at the heel and metatarsal areas; the switches activated at about 70 newtons force.

The walkway, a level tiled floor 7.5 meters long and 2 meters wide, was marked with tape at the beginning and stopping points to provide a zone 6 meters long for monitoring gait. Wires from the various body-mounted

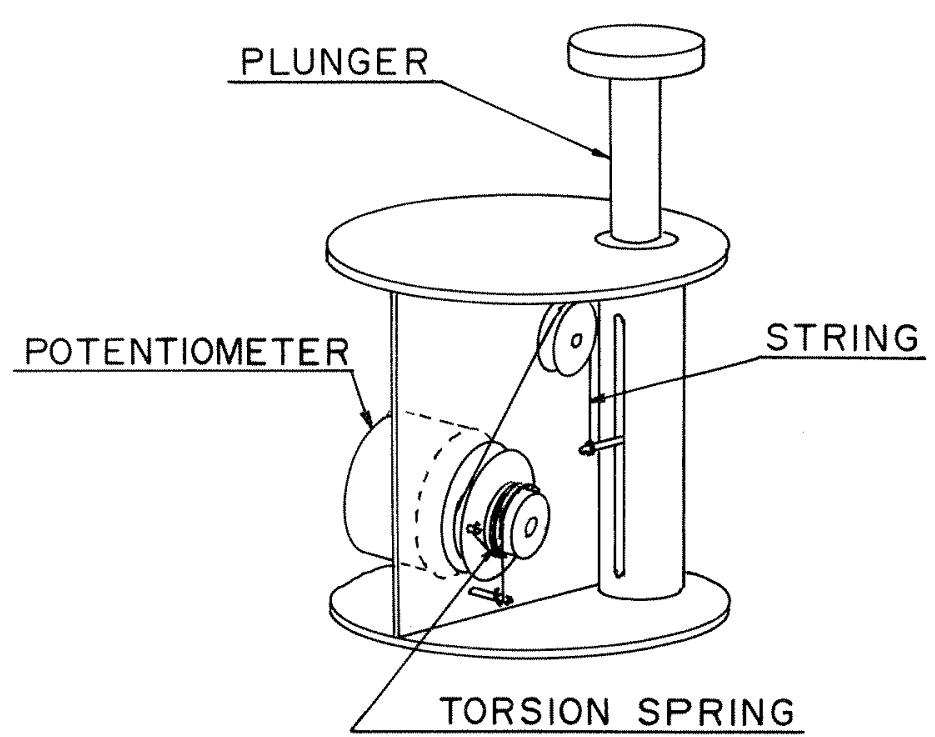

Figure 2.

Axial movement detector.

instruments were plugged into the backpack. Signals from the instruments were conducted to the signal conditioner through a festooned flat multiconductor cable supported by an overhead carrier. The force needed to pull the cable was about one newton. Data signals were conditioned by amplification, filtering with a $33 \mathrm{~Hz}$ roll-off, and conversion from analog-to-digital. Sixteen channels of data were sampled at 60 times per second and stored in the microcomputer and transferred to diskettes for storage and processing.

\section{Data treatment}

The database for each subject consisted of 42 test conditions consisting of the first and second test at the usual, fast, and slow speeds of walking with each of the seven suspension systems. Data collected for each test walk were broken into gait cycles using the beginning of the soundlimb support period as the beginning of a gait cycle. The primary indication for this was peak knee extension confirmed by closure of the heel switch. Gait cycles not included were those clearly accelerative at initiation of gait and decelerative at cessation of gait. See Appendix A for data treatment details. 


\section{RESULTS}

Testing

This study included 20 subjects comprised of 15 males and 5 females. Their ages ranged from 23 to 76 years with an average of 44.5. Residual limb lengths (SL) ranged from 8 to $19 \mathrm{~cm}$ and, when expressed as percent of height $(\% \mathrm{SL} / \mathrm{HT})$, ranged from 4.3 to 10.7 . The shape of the residual limbs (RL) were cylindrical for 10 of the subjects and conical for the rest, and in each of these categories there were six right and four left amputees. Details of subject characteristics may be seen in Appendix B.

The subjects walked at three different speeds nominally defined as "usual," "fast," and "slow." The average velocities were: slow $=0.76$, usual $=0.98$, and fast $=1.23$ meters per second. No distinctions in velocity were noted between subjects of differing SL or shape.

\section{Axial movement (piston action)}

The amount of relative axial movement of the distal aspect of the RL, with respect to the socket, varied among the suspensions and within and among individuals. Differences were noted between the first and second tests within suspensions. The movement averaged $1.91 \mathrm{~cm}(0.75$-inch), and ranged from about 0.6 to about $3.1 \mathrm{~cm}$. The rankings of suspensions by amount of axial movement from least to most were the following:

Cylindrical: RS, SCSP, PTB/WB, ASCW, SC, PTB/C, $\mathrm{PTB} / \mathrm{F} 8$.

Conical: RS, PTB/WB, ASCW, PTB/F8, PTB/C, SCSP, SC.

Mean and standard deviations (SD) of axial movements, in centimeters, may be seen in Figure 3. The axial movement generally decreased with decreasing stump length, but did not correlate well with stump length. Moreover, the movement did not vary as a function of speed of walking.

A weak association existed between the axial excursion and the number of knee waveform deviations on the prosthetic side. The magnitude of the excursion varied directly with the number of deviations for about two-thirds of the subjects. However, when viewed across the total sample, such relations were not distinct. No explanation was found to show why the relation was fairly distinct among some subjects and not among others.

\section{Knee flexion-extension}

The knee flexion-extension waveforms for both the sound and prosthetic sides were examined for deviations. The prosthetic side waveforms contained the higher inci-

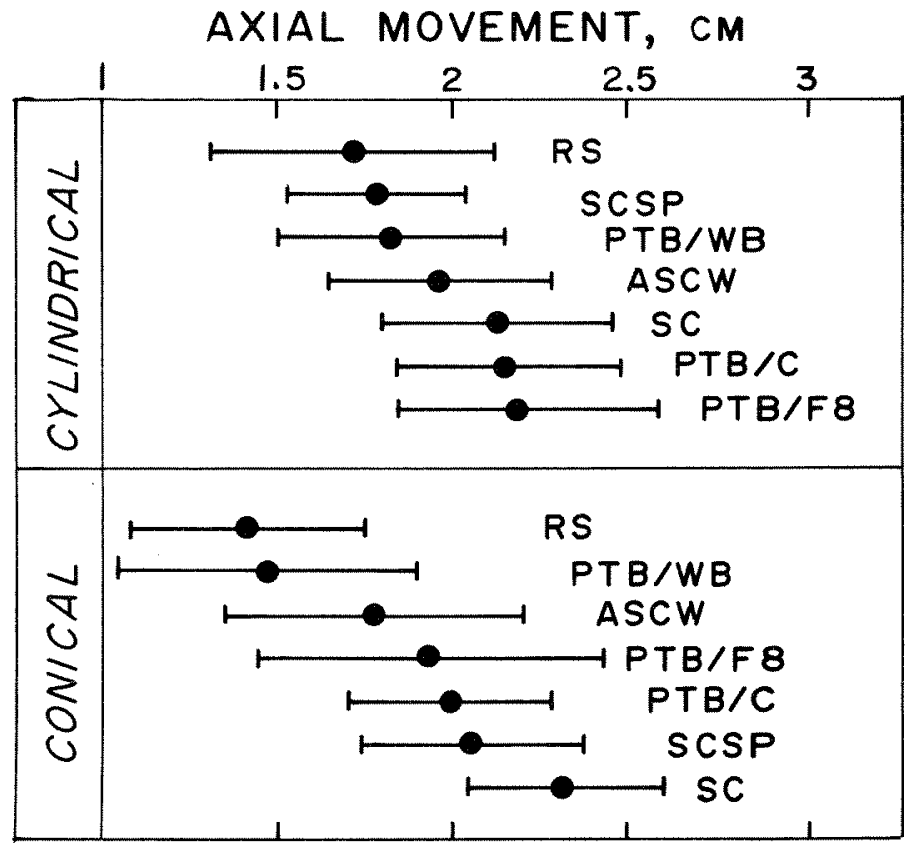

Figure 3.

Axial movement of stump in socket.

dence of deviations. The frequency of these occurrences averaged 17.0 events per gait cycle for the cylindrical shapes, and 14.4 events per gait cycle for the conical. The deviations on the prosthetic side typically consisted of: 1) diminished knee flexion-extension during weightbearing; and, 2) early initiation of swing phase. The number of deviations varied from test to test within, as well as among, individuals. In general, the "fast" walks contained the most deviations, and the "slow" walks the least. The numbers of deviations did not associate with residual limb length, shape, or other physical features noted of the subjects. To simplify comparison of suspensions, the number of events per gait cycle occurring in the first and second test were averaged. The results by suspensions were as follows:

Cylindrical. The average number of deviations ranged from 10.6 with RS, to 23.1 with SC suspension. Ranking the suspensions from the least to the most deviations yielded the following order: RS, ASCW, PTB/WB, SCSP, PTB/F8, $\mathrm{PTB} / \mathrm{C}, \mathrm{SC}$.

Conical. The average number of deviations ranged from 9.8 with the ASCW, to 17.9 with the SC. Ranking the suspensions from the least to the most deviations yielded the following order: ASCW, RS, PTB/WB, SCSP, PTB/C, $\mathrm{PTB} / \mathrm{F} 8, \mathrm{SC}$. The means and SD of knee flexion-extension deviations per gait-cycle by suspension, and by shape of stump, may be seen in Figure 4. 


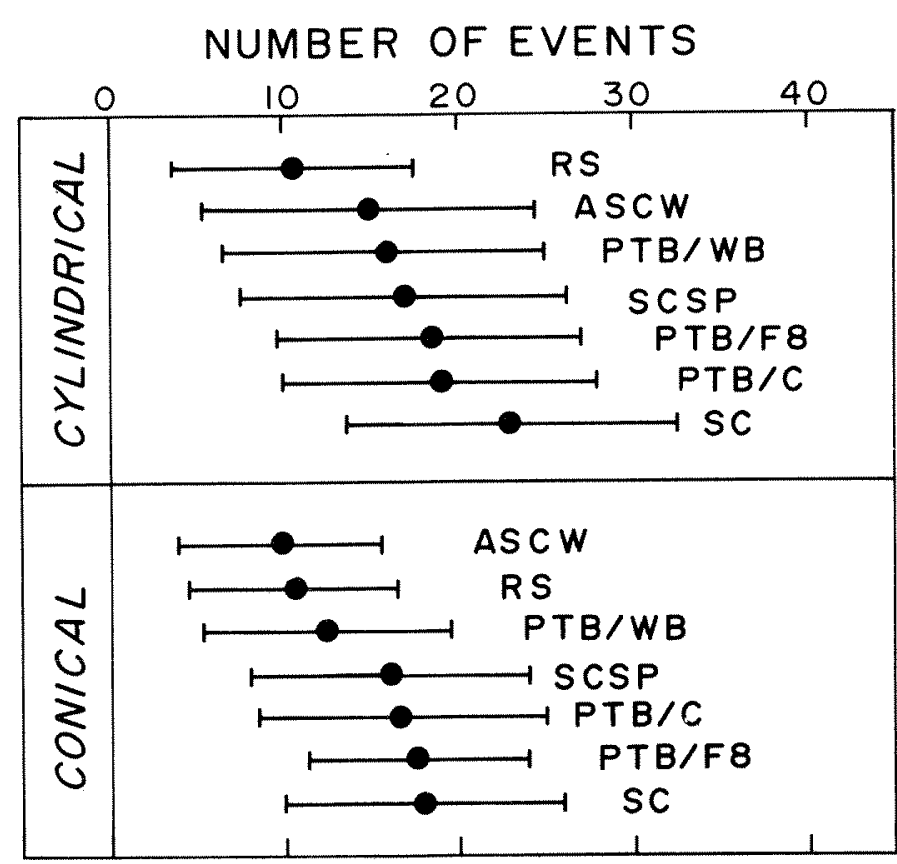

Figure 4.

Numbers of prosthetic side knee flexion-extension deviations per gait cycle.

\section{Harmonic ratios}

These ratios convey an idea of the degree of symmetry in gait; the higher the harmonic ratio, the more symmetric the gait.

The overall average harmonic ratios for the longitudinal acceleration (HACC), vertical acceleration (VACC), and tachometer $(\mathrm{TACH})$, were $1.84,1.77$, and 1.88 , respectively. These values may be seen in Table 1, where results derived from testing 10 normals are included for comparison. It is to be noted that testing of normals included a broad variety of cadences, step lengths, and speeds, some of which were awkward to perform, to provide the comparative base. The harmonic ratios varied among suspensions and related to stump length and shape, but not predictably to speed of walking. Linear regression showed that, almost without exception, the high harmonic ratios were associated with the longer residual limbs. Moreover, the higher harmonic ratios were generally associated with the cylindrical shapes. The HACC harmonic ratio correlated well with stump length, while the VACC and $\mathrm{TACH}$ harmonic ratios correlated modestly. Significances of correlation were $0.054,0.09$, and 0.09 for HACC, VACC, and TACH respectively.
Spectrally, the magnitudes of the Fourier coefficients decreased with increasing harmonics, so that the 12th harmonic represented about 10 percent of the magnitude of the second harmonic. The relation was pronounced between the even and odd harmonics in the range of the fourth to the tenth harmonic, where the odd harmonics were approximately equal to the even harmonics in magnitude.

\section{External work}

The results of external work and efficiencies were supportive of the findings with the primary variables HACC, VACC, and TACH, for purposes of discriminating among different suspensions. However, they were not used in the suspension discrimination procedures, since both work and efficiency were derived from the primary variables.

Waveforms of potential and kinetic energies, plotted as a function of the gait cycle, showed bilateral asymmetries, distortions of the waveforms, presence of higher frequency components, and substantial phase shifts between potential and kinetic energies compared with the performances by normals. Time was not devoted to determine the sources or causes of departures from what might be considered ideal waveforms.

\section{Suspension discrimination}

The technique of using the three-dimensional arrays served to help discriminate performances among the seven different suspension systems. While the AXM did not show a good correlation with length of residual limb, it was included in the discrimination procedure, because it was considered a direct indication of quality of suspension. Iterating, the significances of correlation were $0.054,0.09$, 0.09 and 0.44 for the HACC, VACC, TACH, and AXM, respectively. The resulting weighting factors were: $\mathrm{HACC}$ $=8, \operatorname{VACC}=5, \mathrm{TACH}=5$, and $\mathrm{AXM}=1$.

The influence of the four independent variables,

Table 1.

Mean harmonic ratios for below-knee amputees and for normals.

\begin{tabular}{ccccc}
\hline & HACC & VACC & TACH \\
\hline Amputees Mean & 1.84 & 1.77 & 1.88 \\
& SD & 0.51 & 0.47 & 0.53 \\
Normals & Mean & 4.42 & 3.10 & 4.41 \\
& SD & 1.90 & 1.24 & 1.87 \\
\hline
\end{tabular}

$\mathrm{HACC}=$ Fore-aft acceleration

VACC $=$ Vertical acceleration

$\mathrm{TACH}=$ Tachometer 
WIRTA et al. Analysis of Below-Knee Suspension Systems

Table 2.

Suspensions ranked by weighted normalized best estimates of HACC, VACC, and TACH harmonic ratios and $\mathrm{AXM}$ values.

\begin{tabular}{|c|c|c|c|c|c|c|c|}
\hline$\frac{\text { Cylindrical }}{\% \text { SL/HT }}$ & SCSP & $\mathrm{SC}$ & PTB $/ \mathrm{C}$ & PTB/WB & PTB /F8 & RS & $\mathrm{ASCW}$ \\
\hline 10 & 1.039 & $1.045+$ & $0.931<$ & $0.952<$ & 1.006 & 0.984 & 1.040 \\
\hline 9 & $1.044+$ & 1.016 & $0.936<$ & $0.960<$ & 1.005 & 0.992 & $1.044+$ \\
\hline 8 & $1.050^{+}$ & 0.982 & $0.943<$ & $0.968<$ & 1.003 & 1.003 & $1.048+$ \\
\hline 7 & $1.055+$ & $0.942<$ & $0.952<$ & 0.977 & 1.000 & 1.016 & $1.053+$ \\
\hline 6 & $1.062+$ & $0.893<$ & $0.966<$ & 0.986 & 0.997 & 1.033 & $\underline{1.058}+$ \\
\hline 5 & $1.067+$ & $0.833<$ & 0.987 & 0.996 & 0.994 & $1.057+$ & $1.063+$ \\
\hline$\frac{\text { Conical }}{\% \text { SL/HT }}$ & SCSP & $\mathrm{SC}$ & PTB/C & РТВ/WB & PTB/F8 & RS & ASCW \\
\hline 10 & $0.896<$ & $0.924<$ & $\underline{1.109+}$ & $1.099+$ & 0.945 & $1.109+$ & $0.927<$ \\
\hline 9 & $0.909<$ & $0.912<$ & $1.072+$ & $1.097+$ & 0.964 & $1.088+$ & 0.963 \\
\hline 8 & $0.923<$ & $0.900<$ & 1.031 & $\underline{1.094+}$ & 0.984 & 1.069 & 1.002 \\
\hline 7 & 0.939 & $0.888<$ & 0.988 & $1.091+$ & 1.007 & 1.048 & 1.044 \\
\hline 6 & 0.958 & $0.875<$ & 0.941 & $\underline{1.089+}$ & 1.031 & 1.027 & $1.089+$ \\
\hline 5 & 0.979 & $0.862<$ & $0.888<$ & $1.086+$ & 1.058 & 1.004 & $1.137+$ \\
\hline
\end{tabular}

The better performances are underlined and indicated by a plus sign following the value and the poorer performances are indicated by a less than sign $(<)$ following the value.

weighted as described, resulted in a pair of matrices (one for the cylindrical shapes, and one for the conical), showing the relative ranking of the seven suspension systems with respect to stump length. The scores ranged from 0.833 to 1.045 for the cylindrical, and from 0.862 to 1.109 for the conical: the highest scores represented the best performances. The results may be seen in Table 2 ; the upper quartile values, identified by underlining followed by a plus sign, represent the better scores. The lower quartile values, identified by a following less-than sign $(<)$, represent the poorer scores. The findings are summarized as follows:

Cylindrical. In the upper 25 percent: the SCSP and ASCW for RL lengths from 5 to $9 \% \mathrm{SL} / \mathrm{HT}$; the SC for the longer $(10 \% \mathrm{SL} / \mathrm{HT})$, and RS for the short (5 $\% \mathrm{SL} / \mathrm{HT}$ ). In the lower 25 percent: the PTB/C for stump lengths greater than 5 percent, $\mathrm{SC}$ for stumps shorter than 8 percent, and PTB/WB for stumps longer than 7 percent SL/HT.

Conical. In the upper 25 percent: the PTB/WB for the entire span of lengths, the PTB/C and RS for the long ( $>8 \% \mathrm{SL} / \mathrm{HT}$ ), the ASCW for the short (6 percent and shorter). In the lower 25 percent: the SC for the entire span of lengths, the SCSP for 8 percent and longer, $\mathrm{PTB} / \mathrm{C}$ for the very short, and ASCW for the very long.

\section{Subjective responses}

Some of the subjects in the study provided a number of subjective reactions to the various suspension systems. A digest of comments regarding individual suspension systems included the following:

SCSP: a. Cylindrical: secure; stable

b. Conical: restrictive

SC: $\quad$ Loose; comfortable

PTB/C: Comfortable; slips over knee

PTB/WB: Confident; secure; decreased circulation

PTB/F8: Good for highly active wearer Has better suspension

RS: $\quad$ Sweaty; hot; pulls hair No pistoning

ASCW: Restricts flexion.

The SCSP suspension was the only one that drew differences in comments concerning effects due to the shape of the residual limb. 
Journal of Rehabilitation Research and Development Vol. 27 No. 4 Fall 1990

\section{DISCUSSION}

\section{Laboratory findings}

The ensuing discussion includes a number of terms not in common usage in the prosthetics field. For clarification, the reader is encouraged to refer to the Glossary.

\section{Suspension discrimination}

The rationale for discriminating performances among the seven different suspension systems was to relate characteristics of locomotion to the length and shape of the amputee's residual limb (RL). The criteria were the relative values of four independent variables to answer the question of which suspension accounted for the best gait performance. Three of the variables, most important in discriminating performance, were the harmonic ratios of: 1) longitudinal acceleration; 2) vertical acceleration; and, 3) forward velocity of the body. The fourth independent variable was the axial movement of the distal aspect of the residual limb with respect to the socket. The basic rule was that the highest harmonic ratios and the least axial movement of the stump in the socket constituted the best performance. The magnitude of difference between the various suspensions was relatively small. This was no surprise because all subjects could, in fact, walk with each of the suspensions with no drastic modification in performances.

One of the outstanding findings was the difference in performances attributable to shape of the RL. Subjects with cylindrical shapes did well with the SCSP and ASCW suspensions, and less well with the PTB/WB. In contrast, subjects with conical shapes did well with the PTB/WB and less well with the SCSP. Both of these observations reflect the effect of ratio of soft tissue to bone. The results of this study were based on the comparative performances of only 10 subjects in each of two categories. While the results are believed to be valid, they are not intended to be conclusive.

\section{Kinematics}

The average axial movement at the distal aspect of the $\mathrm{RL}$, sometimes called pistoning, was $1.91 \mathrm{~cm}$. This is in agreement with Burgess et al. (2) who reported threequarters of an inch. The amount of axial displacement at the brim was observed visually, but attempts to measure this displacement accurately were unsuccessful. The reason for the relatively large axial movement developed at the distal aspect of the RL was not determined. It is important to know that such movements occur. Data suggested that slightly less axial movement occurred for the conical- shaped stump than that for the cylindrical stump, which reflected the relative difference in the amount of soft tissue present. Since the amount of axial movement did not relate significantly to speed of walking or to length or shape of $\mathrm{RL}$, it attested to the complex nature of the dynamics of a prosthesis on the residual limb. Differences noted between the first and second test of any given suspension were presumed to reflect variations in the degree of snugness of the suspension, and small changes in stump volume.

Decreased harmonic ratios reflect increased asymmetry in gait. One of the major contributors to asymmetry was the knee flexion-extension pattern on the prosthetic side. Asymmetry resulted from two major causes: 1) diminution of flexion and extension "cushioning" action during the support phase; and, 2) extended duration of the swing phase. First, the amputee restrained knee flexion during the support phase to prevent knee buckling. It was during this part of the gait cycle wherein the incidence of deviations predominated and was common among the subjects. Second, the amputee did not flex and extend the ipsilateral knee during the swing phase as vigorously as on the contralateral side. Compared with the sound side, the swing phase on the prosthetic side was initiated earlier and consequently occupied a larger percentage of the gait cycle. Subjects with conical-shaped residual limbs demonstrated less average numbers of deviations than those with cylindrical shapes. This could be expected because the conical $\mathrm{RL}$ is comprised of less soft tissue than is the cylindrical. During an effort to actively flex and extend the knee, the conical shape provides a firmer coupling to transmit the rotational moment from the bone in the residual limb to the prosthesis. The rubber sleeve suspension resulted in less numbers of deviations in the swing phase compared with the supracondylar, because of the intimacy and resilience of the connection of the prosthesis to the residual limb.

Regarding harmonic analyses, our findings agreed with those reported by Gage (4). The mild suppression of the even harmonics and the substantial elevation of the odd harmonics, Gage suggested, was due to bilateral asymmetries in gait such as unequal step lengths, uneven arm swing, and lateral trunk bending. Robinson et al. (11) stated that normals produced mean harmonic ratios greater than 3.0 and went on to suggest a classification of quality of gait by harmonic ratios: normal greater than 2.0, fair between 1.5 and 2.0 , poor between 1.0 and 1.5 , and very poor less than 1.0. By that scale, the BK amputees in this study demonstrated fair locomotion qualities. Our results also agreed with Robinson's observation that harmonic ratios for the vertical acceleration were lower than those 
for the fore-aft acceleration. In addition, our study showed that the harmonic ratios for the tachometer were in close agreement with the fore-aft acceleration. This should be expected since both instruments sensed performance in the forward direction of progression.

\section{Kinetics}

The mechanical or external work of locomotion and the efficiency of transformation between potential and kinetic energy discriminated performances among the different suspensions. The results using work and efficiency agreed to a great extent with results from the primary variables, HACC, VACC, and TACH. However, work and efficiency were not used in the final discrimination procedures because they were derived from the primary variables.

Of interest were plots of potential and kinetic energies as functions of the gait cycle. Normals' performances revealed relatively smooth curves with potential and kinetic energies phased about 180 degrees apart. Departures from smooth patterns among normals occurred when they walked under conditions alien to their preferred mode, such as very slow walking or awkward relations of cadence to step length. Among amputees, bilateral asymmetries both in the time and displacement domains introduced disturbances which were revealed in potential and kinetic energy waveforms. The wave shapes suggested that the amputees adopted individual neuromuscular control strategies to deal with the constraint imposed by an artificial lower limb. Body movement patterns, sometimes called compensatory movements, were not recorded in this study; therefore, causes for specific types of departures in potential and kinetic energy waveforms were not established.

\section{SUMMARY}

The effect on gait of seven different commonly prescribed BK suspension systems were tested on 20 adult, unilateral amputees who had residual limbs of differing lengths and shapes. The seven suspension systems tested were:

1. Supracondylar-suprapatellar

2. Supracondylar

3. PTB with suprapatellar cuff

4. PTB with suprapatellar cuff and waistbelt

5. PTB with figure-of-eight

6. Rubber sleeve

7. Articulated supracondylar wedge.
Distinctions between different suspension systems were derived from waveform analyses of the forward and vertical accelerations and forward velocity of the body. The technique distinguished differences in performances with the various suspension systems as a function of the length of the RL, and also distinguished whether it was conical or cylindrical in shape. The following observations were noted:

$a$. Test results suggested that the appropriateness of a given suspension system may depend upon the length of the RL, and whether it is cylindrical or conical.

$b$. An average of $1.9 \mathrm{~cm}$ of axial movement of the distal aspect of the RL with respect to the socket was observed. Axial movement was not critical in discriminating among suspension systems. This movement correlated poorly with the length and shape of the RL and was not predictably related to velocity of walking. Less axial movement was noted among subjects with conical than with cylindrical shapes.

c. The knee flexion-extension deviations on the prosthetic side knee movements did not correlate with axial movement of the RL in the socket, or with harmonic ratios. These deviations appeared to contribute to asymmetry of gait.

$d$. Harmonic ratios were derived from accelerometer and tachometer waveforms. These values, which denoted degrees of asymmetry of gait, discriminated between performances with different suspension systems. The higher harmonic ratios (denoting more symmetric gait) associated with the longer RL. Moreover, the higher harmonic ratios were associated with the cylindrical-shaped residual limbs.

\section{ACKNOWLEDGMENT}

We offer special thanks to the San Diego chapter of Amputees in Motion for their enthusiastic community effort in helping recruit subjects for the study. The authors gratefully acknowledge the efforts of Donald Johnson in the collection of test data and for the programming and data processing required in this project.

\section{REFERENCES}

1. Breakey JW: Criteria for the use of supracondylar and supracondylar-suprapatellar suspension in below-knee prostheses. Orthot Prosthet 27(3):14-18, 1973.

2. Burgess EM, Moore JA: A study of interface pressures in the below-knee prosthesis. Bull Prosthet Res 10(28):58-70, 1977. 
3. Cavagna GA, Saibene FP, Margaria R: External work in walking. J Appl Physiol 18(January):1-19, 1963.

4. Gage H: Accelerographic analysis of human gait. ASME Paper 64-WA/HUF-8, 1964.

5. Giacinto JP: The rubber sleeve suspension for below-knee prosthesis. Orthot Prosthet 30(4):17-19, 1976.

6. Girling JG, Cummings G: Artificial limbs fabrication without the use of commercially made components. Prosthet Int 4(2):21-25, 1972.

7. Marschall K, Nitschke R: The PTB prosthesis. Orthot Prosthet Appliance J 22(2):123-126, 1966.
8. Marschall K, Nitschke R: Principles of the patellar tendon supracondylar prosthesis. Orthot Prosthet Appliance J 21(1):33-38, 1969.

9. Pritham CH: Suspension of BK prosthesis: An overview. Orthot Prosthet 33(2):1-19, 1979.

10. Radcliffe CW, Foort JW: The Patella-Tendon Bearing Below-Knee Prosthesis. (Biomechanics Laboratory Report), University of California at Berkeley, 119-130, 1961.

11. Robinson IL, Smidt GL, Arora JS: Accelerographic, temporal, and distance gait factors in below-knee amputees. Phys Ther 57(8):898-904, 1977.

\section{GLOSSARY}

Array. A systematic arrangement of numbers or symbols in tabulated form.

Correlation coefficient. A measurement used to express the degree of association between two variables.

Fourier coefficient. A number prefixed as a multiplier to a given sine or cosine component used in quantitating a periodic waveform. The magnitude denotes the relative contribution of that component to the total waveforms.

Fourier series. The expansion of a periodic function into a series of sines and cosines.

Harmonic. One of the component frequencies characterizing a waveform and is an integral (whole number) multiple of the fundamental frequency. In gait, the fundamental is the stride frequency. Harmonics are integral multiples of the fundamental such as two times, three times, four times, etc.

Harmonic analysis. The analytical procedure of breaking a periodic function into components, each expressed as sine or cosine function and determining the relative contribution of each harmonic to the total waveform.

Harmonic ratio. The harmonic ratio is the quotient derived from dividing the sum of the absolute values of the even harmonics by the sum of the absolute values of the odd harmonics. Applied to this gait study, the higher the harmonic ratio, the more symmetric the gait.

Kinematics. Characterization of motions without consideration of what caused the motions.

Kinetic energy. Energy by virtue of a body in motion. Energy is the capacity to do work.

Kinetics. Consideration of causes of motion, hence the consideration of forces and energies.
Newton. The newton is the amount of force which imparts to a mass of one kilogram an acceleration of one meter per second squared. One newton is approximately 0.225 pounds.

Periodic waveform. A repetitive waveform, such as may be found to recur from gait cycle to gait cycle.

Potential energy. Energy by virtue of position. A raised weight has potential energy.

Regression analysis. A means to determine how two or more variables relate to each other.

Root mean square. The square root of the average of the sums of the squares of a variable. While the root mean square (rms) is almost the same as the arithmetic average, it allows for irregularities, in particular when numbers range above and below zero.

Significance level. Also called the rejection level, and is a value used to judge the probability of two variables not relating to each other. The symbol used to express the probability is " $p$ ". The smaller the " $p$ ", the less likelihood that the relation is pure chance.

Waveform. Shape of a wave as a function of an independent variable such as time, percent gait cycle, or other convenient base.

Weighting function. When some items are not as important as others in a decision-making procedure, the relative importance is through weights assigned to each variable. It may be thought of as being the "voting power" which each variable is given in the process. 


\section{APPENDIX A}

Deviations in knee flexion-extension movements were determined by comparison with normals' waveforms. A study of normals' locomotion showed that Fourier coefficients of knee flexion-extension were related to both velocity and to the ratio of stride frequency to stride length $(\mathrm{F} / \mathrm{L})$. By utilizing functions derived from that study, knee waveforms were synthesized to represent any combination of velocity and $\mathrm{F} / \mathrm{L}$ demonstrated by an amputee. Knee movement comparisons were determined at two percent gait-cycle intervals by subtracting a synthetic waveform from the amputee's wave. A two-SD envelope was provided by the cited study as a function of the gait cycle. If the difference between actual and synthetic waveforms exceeded the two-SD criterion, the event was registered as an anomaly.

Fourier coefficients were calculated for the first 12 harmonics of the fore-aft acceleration (HACC), vertical acceleration (VACC), and tachometer (TACH) waveforms. Coefficients of each harmonic were averaged across the gait cycles within a test walk to represent a test condition.

Harmonic ratios, as described by Robinson et al. (11), were calculated by a procedure consisting of dividing the sum of the even harmonics by the sum of the odd harmonics:

$$
H R=\frac{\Sigma\left(A_{e}+B_{e}\right)}{\Sigma\left(A_{o}+B_{0}\right)}
$$

where:

$$
\mathrm{HR}=\text { harmonic ratio }
$$

$A_{e}, B_{e}=$ absolute values of even harmonics

$A_{0}, B_{0}=$ absolute values of odd harmonics.

Distribution of wave harmonics was facilitated by a normalization technique described by Gage (4). First, the root mean squares (rms) of the coefficients, $C_{n}$, were calculated:

$$
C_{n}=\left[\frac{\left(A_{n}+B_{n}\right)^{2}}{2}\right]^{0.5}
$$

where $A_{n}$ and $B_{n}=$ absolute values of the cosine and sine components for each harmonic $n$. Second, the rms value of each of the 12 harmonics was divided by the value of the second harmonic. As a result, the second harmonic had an arbitrary value of 1.00 and all the rest of the harmonics were some fraction of the second harmonic.

Linear regression was used to relate variables to the shape and length of the residual limb (SL). To make SL comparable among subjects of different stature, the variable was rendered dimensionless by expressing it as a percent of height (\% SL/HT). Slopes and intercepts were used to calculate best estimates of the harmonic ratios and axial movements as functions of SL at $5,6,7,8,9$, and $10 \% \mathrm{SL} / \mathrm{HT}$ to use in suspension discrimina- tion. Correlation coefficients provided the basis for deriving weighting factors.

Whole body external work of locomotion was calculated using the accelerometer and tachometer data in the manner described by Cavagna (3).

\section{Suspension discrimination}

Four independent variables were used to discriminate locomotion performances among the seven suspension systems referenced to the length and shape of the residual limb. The four variables were the following:

1. Harmonic ratios of longitudinal acceleration

2. Harmonic ratios of vertical acceleration

3. Harmonic ratios of forward velocity

4. Axial movement of the residual limb in the socket.

Resolution was achieved by using three-dimensional arrays. A typical array consisted of the following:

$$
\begin{aligned}
& \mathrm{x} \text {-axis }=\text { seven suspensions } \\
& \mathrm{y} \text {-axis }=\text { six lengths in } \% \mathrm{SL} / \mathrm{HT} \\
& \mathrm{z} \text {-axis }=\text { four variables. }
\end{aligned}
$$

Initial filling of the array consisted of calculated harmonic ratios and axial movements as functions of SL by using slope and intercept values derived from regression analyses. To render the different variables to common magnitudes, the values were normalized at each given SL. The criteria for best performances were the highest harmonic ratios and the least axial movement. To make the AXM values consistent in sense with the harmonic ratios, that is, the least AXM representing the best performances, reciprocals of AXM normalized values were entered in the array.

Because all variables did not correlate equally well with SL, devising appropriate weighting factors for each variable was required. These were determined from the significance of correlation. To estimate a gradation of significance, the correlation coefficients and significances were plotted and corresponding estimates of significance were read from the graph. Inverse values of the significances were used as weighting factors for each of the variables as they contributed to the discrimination procedure. The method of applying weighting factors is delineated in the following:

$$
K=\frac{(h H+v V+t T+a A)}{(h+v+t+a)}
$$

where:

$\mathrm{K}=$ relative ranking of the suspension

$\mathrm{H}, \mathrm{V}, \mathrm{T}$, and $\mathrm{A}=$ normalized $\mathrm{HACC}, \mathrm{VACC}, \mathrm{TACH}$, and $\mathrm{AXM}$ $h, v, t$, and $\mathrm{a}=$ weighting. 
Journal of Rehabilitation Research and Development Vol. 27 No. 4 Fall 1990

\section{APPENDIX B}

Characteristics of subjects used in suspension study.

\begin{tabular}{|c|c|c|c|c|c|c|c|c|c|}
\hline Subj & Sex & $\begin{array}{l}\text { Age } \\
\text { yrs }\end{array}$ & $\begin{array}{l}\mathrm{Ht} \\
\mathrm{cm}\end{array}$ & $\begin{array}{l}\text { Wt } \\
\text { kg }\end{array}$ & Shape & $\begin{array}{l}\text { SL } \\
\text { cm }\end{array}$ & Side & $\begin{array}{l}\text { Time } \\
\text { yrs }\end{array}$ & Cause \\
\hline 1 & $\mathrm{~F}$ & 51 & 167 & 67 & cyl & 17 & $\mathrm{R}$ & 1 & Diab \\
\hline 2 & M & 50 & 185 & 136 & cyl & 18 & $\mathrm{R}$ & 2 & Inf \\
\hline 3 & $\mathrm{M}$ & 51 & 177 & 107 & cyl & 17 & $\mathrm{R}$ & 2 & Inf \\
\hline 4 & $\mathrm{M}$ & 32 & 193 & 91 & cyl & 19 & $R$ & 4 & $\operatorname{Tr}$ \\
\hline 5 & $\mathrm{M}$ & 23 & 185 & 104 & cyl & 17 & L & 4 & $\operatorname{Tr}$ \\
\hline 6 & $\mathrm{M}$ & 76 & 178 & 65 & cyl & 15 & L & 3 & AI \\
\hline 7 & $\mathrm{M}$ & 71 & 178 & 74 & cyl & 15 & $\mathrm{~L}$ & 4 & AI \\
\hline 8 & $M$ & 35 & 178 & 77 & cyl & 15 & $\mathrm{R}$ & 1 & CS \\
\hline 9 & $M$ & 35 & 175 & 62 & cyl & 12 & L & 1 & $\mathrm{BD}$ \\
\hline 10 & $\mathrm{M}$ & 37 & 178 & 86 & cyl & 8 & $\mathrm{R}$ & 15 & $\mathrm{Tr}$ \\
\hline 11 & $\mathrm{~F}$ & 62 & 159 & 55 & con & 15 & $\mathrm{R}$ & 7 & $\mathrm{CS}$ \\
\hline 12 & M & 30 & 190 & 82 & con & 17 & L & 4 & $\mathrm{Tr}$ \\
\hline 13 & $\mathrm{M}$ & 41 & 180 & 101 & con & 16 & $\mathrm{R}$ & 30 & Cong \\
\hline 14 & M & 24 & 178 & 75 & con & 15 & $\mathrm{~L}$ & 2 & $\mathrm{Tr}$ \\
\hline 15 & $\mathrm{~F}$ & 40 & 162 & 59 & con & 13 & $\mathrm{R}$ & 2 & $\operatorname{Tr}$ \\
\hline 16 & M & 36 & 188 & 101 & con & 14 & $\mathrm{~L}$ & 6 & $\mathrm{Tr}$ \\
\hline 17 & M & 47 & 188 & 84 & con & 13 & $\mathrm{R}$ & 2 & AI \\
\hline 18 & $\mathrm{~F}$ & 51 & 165 & 65 & con & 11 & $\mathrm{R}$ & 45 & $\operatorname{Tr}$ \\
\hline 19 & $\mathrm{~F}$ & 39 & 166 & 65 & con & 11 & $\mathrm{R}$ & 26 & Cong \\
\hline 20 & M & 58 & 182 & 84 & con & 8 & L & 1 & AI \\
\hline $\begin{array}{l}\text { Time: } \\
\text { Shape: } \\
\text { SL: }\end{array}$ & \multicolumn{9}{|c|}{$\begin{array}{l}\mathrm{Tr}=\text { trauma; } \mathrm{Inf}=\text { Infection; } \mathrm{D} \\
\text { insufficiency; } \mathrm{BD}=\text { Bergers Dis } \\
\mathrm{CS}=\text { Chondro sarcoma } \\
\text { Years since amputation } \\
\text { cyl = cylindrical; con = conical } \\
\text { Residual limb length }\end{array}$} \\
\hline
\end{tabular}

